\title{
The First Online CVIR Case Report Supplement
}

\author{
Ziv J. Haskal · Dierk Vorwerk · Yasuaki Arai
}

Published online: 2 August 2008

(C) Springer Science+Business Media, LLC 2008

As editors of CardioVascular \& Interventional Radiology and fellow clinicians and interventional radiologists, we have made steady and intentional changes over the last several years to increase the quality, standards, and levels of evidence in the journal. This is, after all, the era of evidencebased medicine, when the benefits and risks of treatments we offer should, whenever possible, be based on systematic collection and review of data, and rigorous analysis. This has been a challenging process for Interventional Radiology, a specialty in which the evolution cycles of devices and therapies may be brief and rapid and, even if stable, may not lend themselves to controlled trials because of cohort sizes. Very different than physicians focusing on one disease, we are expert in treating many unique conditions in many small sets. Still, notable areas in our discipline are marked by controlled trials and meta-analyses, such as oncologic interventions, peripheral arterial disease, and portal hypertension. These will naturally grow.

Additionally, CVIR remains equally committed to the value of case-based learning. A succinct report of a single notable case or cases, and perhaps review of pertinent literature, provides an irreplaceable link in the chain of knowledge by heralding a new technique, a warning to others, an extraordinary finding, etc. The case report is also often the natural starting point for those beginning or testing out an academic career.

While we have consciously tightened the acceptance standards for case reports in CVIR, we continue to advocate their importance and relevance. To this end, we announce a new feature this month, the online e-publication of the Case Report Supplement of CVIR. We publish, in one coherent group online, a large set of accepted case reports in our queue. We have organized them to allow readers to sift among the pearls in a more organ- and disease-based fashion. Like any print publication, they bear full online citations and credit.

You will find the online supplement on CVIR's homepage; go to www.springer.com/270 and click on Online Version Available to view the entire Table of Contents and the link to this special supplement.

\footnotetext{
Z. J. Haskal ( $₫)$

Division of Vascular and Interventional Radiology,

New York-Presbyterian Hospital/Columbia Univ. Medical Ctr., 177 Fort Washington Ave., MHB 4-100, New York, NY, USA e-mail: zh50@columbia.edu

D. Vorwerk

Department of Radiology, Klinikum Ingolstadt,

Krumenauerstrasse 25, 85049 Ingolstadt, Bavaria, Germany

e-mail: dierk.vorwerk@klinikum-ingolstadt.de

Y. Arai

Division of Diagnostic Radiology and Nuclear Medicine,

National Cancer Hospital, 5-1-1 Tsukiji, Chuo-ku,

Tokyo 104-0045, Japan

e-mail: arai-y3111@mvh.biglobe.ne.jp
} 